\title{
Comparative Oral Absorption of Different Citicoline and Homotaurine Formulations: A Single-Dose, Two-Period Crossover Trial in the Dog
}

\author{
Andrea Marchegiani ${ }^{1}$ ( ${ }^{\text {, Ferdinando Nicoletti }}{ }^{2,3}$, Maria Rosaria Romano², Decio Capobianco ${ }^{4}$, \\ Ciro Costagliola ${ }^{5}$, Carlotta Marini ${ }^{1}$, Giuseppe Lubrano Lavadera ${ }^{6}$, Roberto Ciccocioppo ${ }^{7}$, \\ Andrea Spaterna ${ }^{1}$
}

\footnotetext{
${ }^{1}$ School of Biosciences and Veterinary Medicine, University of Camerino, Camerino, Italy; ${ }^{2}$ IRCCS Neuromed, Pozzilli, Italy; ${ }^{3}$ Department of Physiology and Pharmacology, Sapienza University of Rome, Rome, Italy; ${ }^{4}$ Glaucoma Service, Ophthalmic Outpatients Department, ASL NA1 and CE, Naples, Italy; ${ }^{5}$ Department of Medicine and Health Sciences "V. Tiberio", University of Molise, Campobasso, Italy; ${ }^{6}$ Department of Economics and Statistics, DISES, University of Salerno, Salerno, Italy; ${ }^{7}$ School of Pharmaceutical Sciences and Health Products, University of Camerino, Camerino, Italy
}

Correspondence to: Andrea Marchegiani, andrea.marchegiani@unicam.it Keywords: Citicoline, Homotaurine, Bioavailability, Neurodegenerative Diseases, Ophthalmological Diseases, Glaucoma Received: June 13, $2019 \quad$ Accepted: July 21, $2019 \quad$ Published: July 24, 2019

Copyright $\odot 2019$ by author(s) and Scientific Research Publishing Inc. This work is licensed under the Creative Commons Attribution International License (CC BY 4.0). http://creativecommons.org/licenses/by/4.0/

\section{(c) (i) Open Access}

\section{ABSTRACT}

Background: Citicoline and homotaurine are compounds with a potent neuroprotective activity and they have been administered for many years in the treatment of numerous neurodegenerative and ophthalmological diseases, including glaucoma. Initially available only as liquid form, through parenteral route, nowadays citicoline can be administered also as tablet but no data on bioavailability of these different forms are available. In the present study, pharmacokinetics of citicoline in tablet versus vials, each at the therapeutic dose of $500 \mathrm{mg}$, in addition to $50 \mathrm{mg}$ of homotaurine was investigated. Materials and methods: Ten mixed breed dogs received a single dose of $50 \mathrm{mg}$ oral homotaurine and $500 \mathrm{mg}$ citicoline in tablet and vials with the same dose were administered after a seven days wash-out period. Parameters assessed for citicoline metabolites (cytidine, uridine and choline) were $\mathrm{AUC}_{0-\mathrm{t}}$, $C_{\max }$ and $T_{\max }$. Results: Citicoline bioavailability appeared to be slightly higher for the tablet compared to the vial formulation. Cytidine is equivalent in absorption dynamics both for tablet and liquid form; uridine for tablet reaches its maximum and is reabsorbed more quickly while choline for the liquid form reaches the maximum first and is reabsorbed more quickly. Conclusions: Citicoline in tablet and liquid formulation have pharmacokinetic properties leading to a very similar bioavailability. 


\section{INTRODUCTION}

Citicoline and homotaurine are compounds endowed with potent neuroprotective activity [1]. Citicoline (cytidine (5') diphosphocholine) and its hydrolysis products, cytidine and choline, play an important role in generation of phospholipids involved in membrane formation and contribute to critical metabolic functions such as formation of nucleic acids, proteins, and acetylcholine [2]. Additionally, exogenously administered citicoline prevents, reduces, or reverses the effects of ischemia and/or hypoxia in several animal and cellular models [2] and they may alleviate free fatty acid-induced toxicity, which generally accompanies ischemic insult $[3,4]$. The effects attributed to citicoline include antiapoptotic effects, neurotrophic properties, protection after partial optic nerve crush, reduction of excitotoxicity, effects on nonglutamatergic neurotransmitter systems, and effects on remyelination [5]. In addition, citicoline appears to be a promising agent for the improvement of cognitive impairment [6].

In recent years, a large body of research has been addressed to identify neuroprotective drugs and food supplements able to prevent neurodegenerative pathologies and preserve visual function and these efforts revealed how the use of a combination of compounds with synergistic multitarget effects might offer a significant approach for disease prevention, including neurodegenerative pathologies and retinal neurodegeneration $[1,7-9]$.

Homotaurine (3-aminopropanesulfonate), an analogue of 4 -aminobutyrate ( $\gamma$-aminobutyric acid, GABA), is a small natural aminosulfonate compound identified in different species of marine red algae (Acrosorium uncinatum, Cladophora densa, Chondrus ocellatus, Gratelupia livida and Rhodymenia palmataa) and then chemically synthesized under the name of tramiprosate [10]. This substance may interfere with several cellular pathways and exert neuroprotective and neurotropic activities through different mechanisms including effects against the oxidative damage to DNA, antifibrillogenic activity, and antinociceptive and analgesic activities, related to the activation of GABA type-A receptors. In addition, homotaurine prevents the neurotoxicity of $\mathrm{A} \beta$ peptide by reducing amyloid aggregation in humans $[11,12]$.

For its nootropic abilities, citicoline has been used for many years in the treatment of numerous neurodegenerative diseases and, more recently, in ophthalmology as an adjuvant in the treatment of glaucoma and amblyopia [13-15]. While homotaurine is available only in solid form, citicoline is used for oral administration in liquid or solid formulation depending on the preferences of the patient or the prescriber.

The aim of this study was to compare the plasma levels of citicoline of two different commercially available formulations of tablet (homotaurine included in this formulation) and vials (homotaurine administered in combination) in adult dogs after a single $500 \mathrm{mg}$ oral dose, the dose normally recommended for these products.

\section{METHODS}

The study was designed as a single-dose, two-period crossover trial. All animals-related procedures described were conducted in accordance with the recommendation of the Italian Ethical Committee and in accordance to the European legislations on animal handling (European Parliament Directive 2010/63/EU). Informed consent form was obtained from all owners participating in the study.

\subsection{Subject Population and Investigated Products}

Ten mixed breed dogs (males and females equally distributed, mean age 5 years, weight $8-10 \mathrm{~kg}$ ) suffering from spontaneous neurological pathology were enrolled in the study. Housing conditions were maintained on a consistent basis during the length of the study and all dogs were kept on the same diet throughout the entire experimental period. The comparative oral absorption study was conducted under fasting conditions as this is considered to be the most sensitive condition to detect a potential difference between formulations [16].

In all dogs, a single dose of $500 \mathrm{mg}$ citicoline and $50 \mathrm{mg}$ oral homotaurine formulated in the same tablet was administered. After a wash-out period of seven days, a single liquid dose of $500 \mathrm{mg}$ citicoline 
and $50 \mathrm{mg}$ homotaurine was administered in the same dogs.

\subsection{Sample Collection and Plasma Separation}

For the assessment of oral absorption of citicoline, blood aliquots necessary for the determination of bioavailability were obtained in concomitance with the normal monitoring procedure of the neurological pathologies, which include complete blood count, serum biochemistry, daily blood glucose curves. Each pre-dose blood sample $(1 \mathrm{ml})$ was collected within 5 minutes before dosing. The post-dose sample $(1 \mathrm{ml})$ were collected at $0.5,1,1.5,2,3,4,6,8,10,12$ and 24 hours after dosing into vacuum tubing containing $\mathrm{K}_{2}$ EDTA. No extra blood samples were collected for repeat laboratory tests.

After collection, all blood samples were stored and transferred in a container precooled with refrigerant gel packs and subsequently centrifuged at $1500 \mathrm{rpm}$ at $4^{\circ} \mathrm{C}$ for $10 \mathrm{~min}$ within $10 \mathrm{~min}$ of collection. After centrifugation, the separated plasmas were transferred into suitably labelled polypropylene tubes and stored at $-20^{\circ} \mathrm{C}$ until the time of the determinations.

\subsection{Analytical Methods}

Pharmacokinetic parameters

Primary endpoint included comparisons of the plasma levels of citicoline of the two different commercially available formulations. The following parameters were assessed: area under the concentration curve $\left(\mathrm{AUC}_{0-\mathrm{t}}\right)$ at the last measurement $(24 \mathrm{~h})$ estimated using trapezoid rule; maximum concentration level $\left(C_{\max }\right)$, time of maximum concentration $\left(T_{\max }\right)$ and their standard deviations. The AUC is the most reliable measure of the biological availability as it measures the entire response over time and provides a more accurate picture of bioavailability while the $C_{\max }$ represents only one point in time and is therefore less robust [17].

Secondary endpoint included the evaluation of differences between the two formulations, using $\mathrm{f}$ test for and $C_{\max }$ from the ANOVA estimate. Exact Wilcoxon-Mann-Whitney Test for $T_{\max }$ was implemented.

Evaluation of oral absorption of different citicoline formulation was performed through comparison of its metabolites choline, cytidine, and uridine in each investigated product.

Pharmacokinetic parameters including $C_{\max }, \mathrm{AUC}_{0-\mathrm{t}}$ and $T_{\max }$ for all two investigational product groups were generated using R CORE TEAM, 2017 and values with $\mathrm{p}<0.05$ were considered statistically significant.

Choline spectrophotometry evaluation

For free choline, the Choline/Acetylcholine Quantification Kit from Sigma Aldrich was applied directly to the plasma. This kit includes buffer solution, choline enzyme mix, acetylcholinesterase, choline standard and choline probe; it was stored at $-20^{\circ} \mathrm{C}$ until analysis. The choline enzyme mix was reconstituted with $220 \mu \mathrm{l}$ of buffer, while the concentrated solution of choline was prepared by reconstituting with $100 \mu \mathrm{l}$ of buffer the standard choline $(50 \mathrm{mM})$. For the colorimetric analysis $10 \mu \mathrm{l}$ of the concentrated solution of choline were dissolved in $990 \mu \mathrm{l}$ of buffer, thus obtaining the standard solution of choline $0.5 \mathrm{mM}$.

The colorimetric evaluation of free choline using the Choline/Acetylcholine Quantification Kit was carried out with the Shimadzu UV-Mini 1240 spectrophotometer, using the UV Brand micro-rain. 0, 2, 4, 6,8 and $10 \mu \mathrm{l}$ of the standard solution of choline were dosed into the micro cuvette and brought to the final volume of $50 \mu \mathrm{l}$, to obtain respectively standards of $1,2,3,4$ and $5 \mathrm{nM} /$ well and a white. $50 \mu \mathrm{l}$ of sample were dosed respectively as the standards within the microcuvette. For the determination of free choline, a reaction mix was prepared containing: $46 \mu \mathrm{l}$ of buffer, $2 \mu \mathrm{l}$ of choline probe and $2 \mu \mathrm{l}$ of choline enzyme mix, for each sample and standard. After 30 minutes of reaction and mild stirring by the addition of the reaction mix, the samples and standards were read at the spectrophotometer at a wavelength of 570 $\mathrm{nm}$. By reading the standards, the calibration line was created, from which the concentration of free choline in the samples was then identified.

Cytidine and uridine HPLC evaluations

Cytidine and uridine were assessed with HPLC RP chromatography, which was performed with col- 
umn C18 and mobile phase consisting of solutions A: 0.1 M phosphate buffer $\mathrm{pH} 5.0$ (monobasic potassium phosphate, adjusted with potassium hydroxide); B: acetonitrile. The chromatographic essay was run with a constant flow of $1 \mathrm{ml} /$ minute, according to the gradient, as shown in Table 1.

The total duration of the run was 25 minutes, including the reconditioning time of the column. The reading wavelength for cytidine and uridine was $254 \mathrm{~nm}$.

Concentrated solutions and standard solutions for the calibration straight lines were prepared by dissolving the nucleotides in $0.1 \mathrm{M}$ phosphate buffer at $\mathrm{pH} 5.0$ (solution $\mathrm{A}$ ).

Cytidine has a retention time of 4.66 minutes, while uridine is 5.6 minutes. For the preparation of the samples for HPLC RP analysis of Cytidine and Uridine, the following reagents were used: $65 \%$ perchloric acid (Carlo Erba Reagents), Monobasic Potassium Phosphate (Sigma Aldrich), Potassium Hydroxide in pellets (Carlo Erba Reagents).

For the realization of the mobile phases the following reagents were used: Acetonitrile HPLC grade (Carlo Erba Reagents) and Monobasic Potassium Phosphate (Sigma Aldrich). The analytical standards of Cytidine and Uridine were obtained from Sigma Aldrich. For the HPLC analysis, the YL-Instrument chromatographic system was set up with a C18 Mediterranean column $(250 \mathrm{~mm} \times 4.6 \mathrm{~mm}, 5 \mu \mathrm{m})$, a precolumn guard filter, a UV detector and a manual injector. To purify the samples, a perchloric acid deproteinization was performed, using $200 \mu \mathrm{l}$ of $2 \mathrm{M}$ perchloric acid, added to $200 \mu \mathrm{l}$ of plasma. The solution was left to react for at least 10 minutes at $0-4^{\circ} \mathrm{C}$ and, subsequently, was centrifuged at $5000 \mathrm{rpm}$ for $15 \mathrm{~min} .300 \mu \mathrm{l}$ of supernatant were recovered, to which $110 \mu \mathrm{l}$ of $4 \mathrm{M}+\mathrm{KH}_{2} \mathrm{PO}_{4} 1 \mathrm{M} \mathrm{KOH}$ buffer solution (11.22 $\mathrm{g}$ of $\mathrm{KOH}+6.8 \mathrm{~g}$ of $\mathrm{KH}_{2} \mathrm{PO}_{4}$ in $50 \mathrm{ml}$ ) were added. The sample solution so treated was left to rest for at least 10 minutes at $0-4^{\circ} \mathrm{C}$, and then it was finally centrifuged again at $5000 \mathrm{rpm}$ for 10 $\min$.

\section{RESULTS}

This study involved a single dose, two period cross-over evaluation to assess the single-dose (500 $\mathrm{mg}$ ) oral bioequivalence of two citicoline formulations in $10 \mathrm{dogs}$ under fasting conditions. No adverse effects were reported by the subjects taking any of these products.

Pharmacokinetic parameters of citicoline metabolites concentrations by mean AUC and standard deviation, $C_{\max }$ and $T_{\max }$ for each time interval and formulation at the different time intervals up to $24 \mathrm{~h}$ are given in Tables 2-4. Summary of pharmacokinetic parameters is reported in Table 5.

The graphical representations of the mean concentrations of cytidine, uridine and choline for the two formulations as a function of time are presented in Figures 1-3.

Cytidine and choline plasma exposure for tablet was slightly higher than that for liquid form, while uridine plasma exposure was slightly higher for liquid form; however, no statistical differences were observed for plasma exposure between solid and liquid formulation.

The tablet formulation yielded earlier plasma peak concentrations for uridine (5.1 hours) but not for

Table 1. HPLC RP parameters.

\begin{tabular}{cccc}
\hline time (minutes) & A\% & B\% & Flow ml/minute \\
\hline 0 & 97 & 3 & 1 \\
7 & 97 & 3 & 1 \\
7.01 & 50 & 50 & 1 \\
15 & 50 & 50 & 1 \\
16 & 97 & 3 & 1 \\
\hline
\end{tabular}


Table 2. Summary of pharmacokinetic parameters for Cytidine.

\begin{tabular}{|c|c|c|c|c|}
\hline Variable & Tablet & Liquid & Test Score ${ }^{\star}$ & $p$ values \\
\hline $\mathrm{AUC}_{0-\mathrm{t}}^{1} \quad(\mathrm{SD})$ & $952.22(153.34)$ & $942.55(148.87)$ & 0.114 & 0.744 \\
\hline$C_{\max }^{3}(\mathrm{SD})$ & $63.27(9.67)$ & $59.667(8.522)$ & 0.772 & 0.405 \\
\hline$T_{\max }^{4}(\mathrm{SD})$ & $1.500(1.08)$ & $1.300(0.675)$ & 0.000 & 1.000 \\
\hline
\end{tabular}

Abbreviations: $\mathrm{AUC}_{0-\mathrm{t}}$, area under the concentration curve until $24 \mathrm{~h}$ units are $\mu \mathrm{mol} / \mathrm{L} \cdot \mathrm{hr} ; C_{\max }$, maximum concentration units are $\mu \mathrm{mol} / \mathrm{L} ; T_{\max }$, time of maximum concentration $(\mathrm{hr}) .{ }^{\star} \mathrm{F}$ score obtained from ANOVA 90\% Confidence intervals Exact Wilcoxon-Mann-Whitney Test for $T_{\max }$.

Table 3. Summary of pharmacokinetic parameters for Uridine.

\begin{tabular}{|c|c|c|c|c|}
\hline Variable & Tablet & Liquid & Test Score ${ }^{*}$ & $p$ values \\
\hline $\mathrm{AUC}_{0-\mathrm{t}}^{1} \quad(\mathrm{SD})$ & $252.381(56.895)$ & $269.981(95.203)$ & 0.291 & 0.604 \\
\hline$C_{\max }^{3}(\mathrm{SD})$ & $20.80(7.827)$ & $22.34(8.275)$ & 0.199 & 0.668 \\
\hline$T_{\max }^{4}(\mathrm{SD})$ & $5.100(3.071)$ & $5.400(2.757)$ & -0.216 & 0.897 \\
\hline
\end{tabular}

Abbreviations: $\mathrm{AUC}_{0-\mathrm{t}}$, area under the concentration curve until $24 \mathrm{~h}$ units are $\mu \mathrm{mol} / \mathrm{L} \cdot \mathrm{hr} ; C_{\max }$, maximum concentration units are $\mu \mathrm{mol} / \mathrm{L} ; T_{\max }$, time of maximum concentration $(\mathrm{hr}) .{ }^{\star} \mathrm{F}$ score obtained from ANOVA 90\% Confidence intervals Exact Wilcoxon-Mann-Whitney Test for $T_{\max }$.

Table 4. Summary of pharmacokinetic parameters for choline.

\begin{tabular}{|c|c|c|c|c|}
\hline Variable & Tablet & Liquid & Test Score* & $p$ values \\
\hline $\mathrm{AUC}_{0-\mathrm{t}}^{1}(\mathrm{SD})$ & $72.199(11.41)$ & $69.941(11.36)$ & 0.329 & 0.582 \\
\hline$C_{\max }^{3}(\mathrm{SD})$ & $4.23(1.04)$ & $4.15(0.88)$ & 0.057 & 0.818 \\
\hline$T_{\max }^{4}(\mathrm{SD})$ & $2.10(0.52)$ & $2.05(0.37)$ & 0.000 & 1.000 \\
\hline
\end{tabular}

Abbreviations: $\mathrm{AUC}_{0-\mathrm{t}}$, area under the concentration curve until $24 \mathrm{~h}$ units are $\mu \mathrm{mol} / \mathrm{L} \cdot \mathrm{hr} ; C_{\max }$, maximum concentration units are $\mu \mathrm{mol} / \mathrm{L} ; T_{\max }$, time of maximum concentration (hr). ${ }^{\star} \mathrm{F}$ score obtained from ANOVA 90\% Confidence intervals Exact Wilcoxon-Mann-Whitney Test for $T_{\max }$.

Table 5. Summary of pharmacokinetics parameters.

\begin{tabular}{ccccccc}
\hline & \multicolumn{2}{c}{ Cytidine } & \multicolumn{2}{c}{ Uridine } & \multicolumn{2}{c}{ Choline } \\
\hline Variable & Tablet & Liquid & Tablet & Liquid & Tablet & Liquid \\
\hline AUC $_{0-\mathrm{t}}$ & 952.22 & 942.55 & 252.381 & 269.981 & 72.199 & 69.941 \\
$(\mathrm{SD})$ & $(153.34)$ & $(148.87)$ & $(56.895)$ & $(95.203)$ & $(11.41)$ & $(11.36)$ \\
$C_{\max }(\mathrm{SD})$ & $63.27(9.67)$ & $59.667(8.522)$ & $20.80(7.827)$ & $22.34(8.275)$ & $4.23(1.04)$ & $4.15(0.88)$ \\
$T_{\max }(\mathrm{SD})$ & $1.500(1.08)$ & $1.300(0.675)$ & $5.100(3.071)$ & $5.400(2.757)$ & $2.10(0.52)$ & $2.05(0.37)$ \\
\hline
\end{tabular}




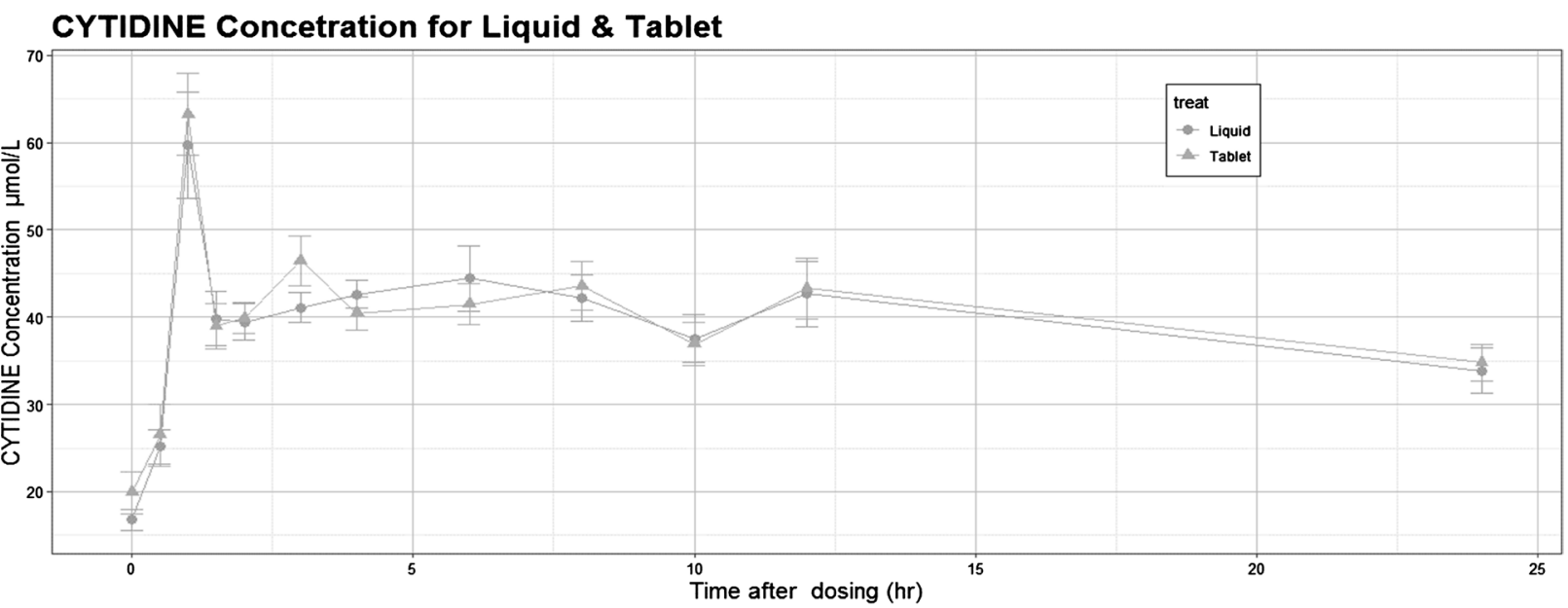

Figure 1. Area under the concentration curve (AUC) of Cytidine for liquid and tablet administration) estimated using trapezoid rule (R CORE TEAM, 2017).

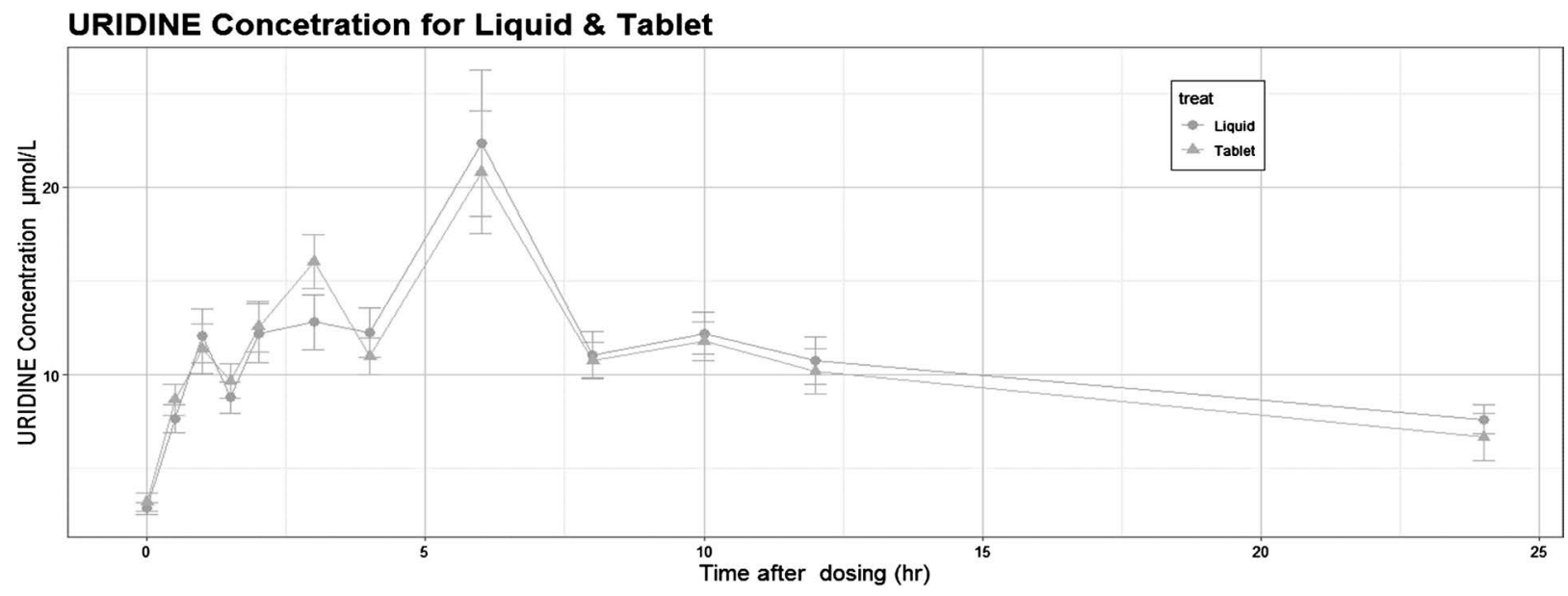

Figure 2. Area under the concentration curve (AUC) of Uridine for liquid and tablet administration) estimated using trapezoid rule ( $R$ CORE TEAM, 2017).

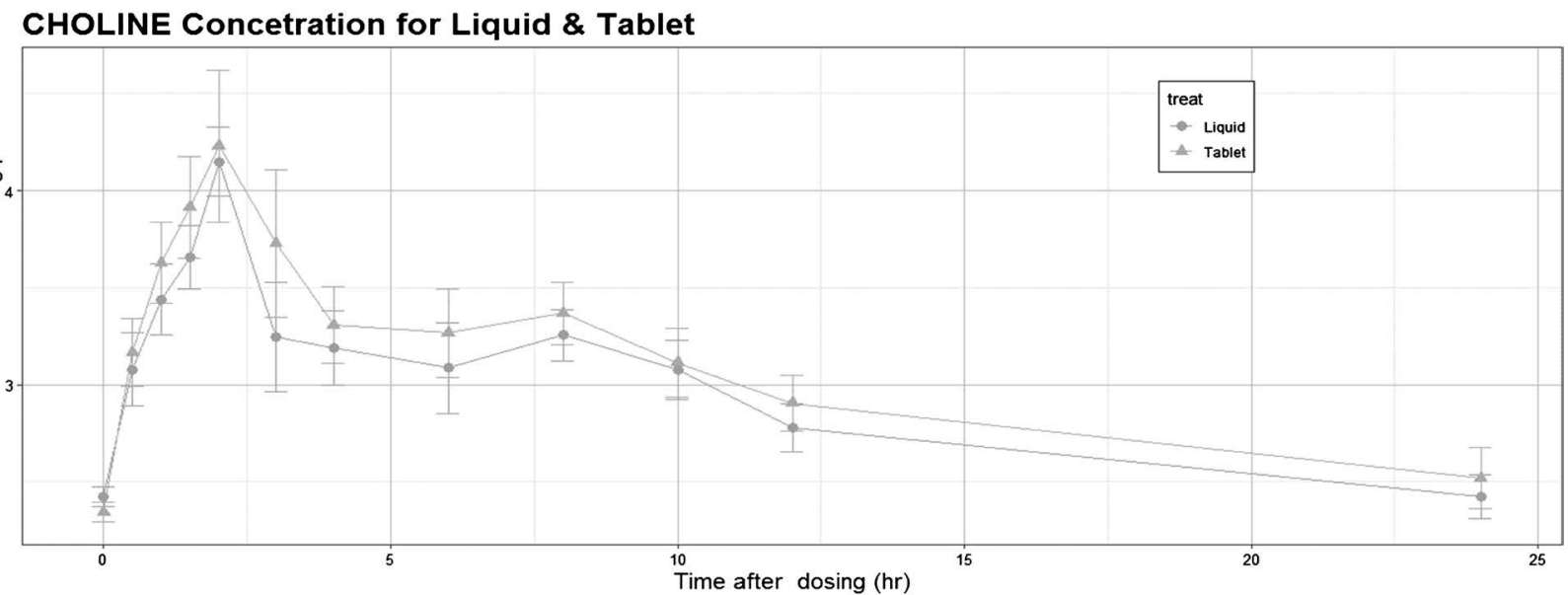

Figure 3. Area under the concentration curve (AUC) of Choline for liquid and tablet administration estimated using trapezoid rule ( $R$ CORE TEAM, 2017). 
cytidine and choline. $C_{\max }$ was higher for both cytidine $(63.27 \mu \mathrm{mol} / \mathrm{L})$ and choline $(4.23 \mathrm{ng} / \mathrm{ng} / \mu \mathrm{l})$ but not for uridine $(20.80 \mu \mathrm{mol} / \mathrm{L})$.

The liquid formulation yielded earlier peak plasma concentrations for both cytidine (1.3 hours) and choline $(2.05$ hours $) . C_{\max }$ was higher for uridine $(22.34 \mu \mathrm{mol} / \mathrm{L})$, but not for cytidine $(59.67 \mu \mathrm{mol} / \mathrm{L})$ and choline $(4.15 \mathrm{ng} / \mu \mathrm{l})$.

The results from the $\mathrm{f}$ test for $\mathrm{AUC}_{0-\mathrm{t}}$ and $C_{\max }$ and of the Exact Wilcoxon-Mann-Whitney Test for $T_{\max }$ do not show any significant difference.

\section{DISCUSSION}

Bioequivalence of different compound formulations were made by comparing pharmacokinetic parameters such as $C_{\max }, T_{\max }$ and AUC $[18,19]$.

Typically, bioequivalence study are basically based on comparative bioavailability study designed to establish equivalence between test and reference products [16]. Bioavailability comparisons are based on plasma concentrations of the parent compound hence for compounds that have a relatively short half-life, undetectable plasma levels, or known metabolites that affect efficacy and/or toxicity, while assessments based on the parent compound alone may be misleading [16]. For the purpose of the study, since citicoline metabolism yields three major metabolites as cytidine, uridine and choline, we extended our comparisons of $C_{\max }, T_{\max }$ and AUC to include all of them.

Cytidine is equivalent in absorption dynamics both for tablet and liquid form; but in the case of uridine the tablet seems to reach its maximum and is reabsorbed more quickly. Finally, the choline has exactly the opposite effect; the liquid version reaches the maximum first and is reabsorbed more quickly.

Cytidine and choline metabolites evaluations show similar paths for tablet and liquid forms, in terms of $\mathrm{AUC}_{0-\mathrm{t}}, C_{\max }$ and $T_{\max }$ and relative statistical test do not show any significant difference. Uridine PK evaluation shows differences statistically significant regarding the absorption in the long period.

For a decision of the Authors, homotaurine tablet was administered in combination with citicoline vials to eliminate possible interferences during bioequivalence assessment between solid and liquid form, thus making the administration homogeneous in both groups of dogs evaluated.

Pharmacokinetics assessment of citicoline was feasible regardless of homotaurine and its metabolites in plasma. Further studies evaluating bioavailability of solely administered citicoline would be desirable.

\section{CONCLUSIONS}

Results of the present trial suggest that citicoline in tablet and liquid formulation have similar pharmacokinetics properties leading to a bioequivalence of their metabolites.

To our knowledge, this is the first trial aimed to the evaluation of bioequivalence of citicoline in tablet versus vial formulations carried out in both human and veterinary medicine, providing additional insights regarding the bioequivalence of tablet and liquid formulations in future citicoline trials.

\section{FUNDING}

This research did not receive any specific grant from funding agencies in the public, commercial, or not-for-profit sectors.

\section{CONFLICTS OF INTEREST}

The authors report no conflicts of interest in this work. This research did not receive any specific grant from funding agencies in the public, commercial, or not-for-profit sectors 1 .

\section{REFERENCES}

1. Davinelli, S., Chiosi, F., Di Marco, R., Costagliola, C. and Scapagnini, G. (2017) Cytoprotective Effects of Citi- 
coline and Homotaurine against Glutamate and High Glucose Neurotoxicity in Primary Cultured Retinal Cells. Oxidative Medicine and Cellular Longevity, 2017, Article ID: 2825703. https://doi.org/10.1155/2017/2825703

2. Bruhwyler, J., Liégeois, J.F. and Géczy, J. (1998) Facilitatory Effects of Chronically Administered Citicoline on Learning and Memory Processes in the Dog. Progress in Neuro-Psychopharmacology and Biological Psychiatry, 22, 115-128. https://doi.org/10.1016/S0278-5846(97)00183-8

3. D’Orlando, K.J. and Sandage, B.W.J. (1995) Citicoline (CDP-Choline): Mechanisms of Action and Effects in Ischemic Brain Injury. Neurological Research, 17, 281-284. https://doi.org/10.1080/01616412.1995.11740327

4. Weiss, G.B. (1995) Metabolism and Actions of CDP-Choline as an Endogenous Compound and Administered Exogenously as Citicoline. Life Sciences, 56, 637-660. https://doi.org/10.1016/0024-3205(94)00427-T

5. Grieb, P. (2014) Neuroprotective Properties of Citicoline: Facts, Doubts and Unresolved Issues. CNS Drugs, 28, 185-193. https://doi.org/10.1007/s40263-014-0144-8

6. Gareri, P., Castagna, A., Cotroneo, A.M., Putignano, S., De Sarro, G. and Bruni, A.C. (2015) The Role of Citicoline in Cognitive Impairment: Pharmacological Characteristics, Possible Advantages, and Doubts for an Old Drug with New Perspectives. Clinical Interventions in Aging, 10, 1421-1429. https://doi.org/10.2147/CIA.S87886

7. Davinelli, S., Sapere, N., Visentin, M., Zella, D. and Scapagnini, G. (2013) Enhancement of Mitochondrial Biogenesis with Polyphenols: Combined Effects of Resveratrol and Equol in Human Endothelial Cells. Immunity \& Ageing, 10, 28. https://doi.org/10.1186/1742-4933-10-28

8. Davinelli, S., Calabrese, V., Zella, D. and Scapagnini, G. (2014) Epigenetic Nutraceutical Diets in Alzheimer's Disease. The Journal of Nutrition, Health \& Aging, 18, 800-805. https://doi.org/10.1007/s12603-014-0552-y

9. Davinelli, S., Maes, M., Corbi, G., Zarrelli, A., Willcox, D.C. and Scapagnini, G. (2016) Dietary Phytochemicals and Neuro-Inflammaging: From Mechanistic Insights to Translational Challenges. Immunity \& Ageing, 13, 16. https://doi.org/10.1186/s12979-016-0070-3

10. Martorana, A., Di Lorenzo, F., Manenti, G., Semprini, R. and Koch, G. (2014) Homotaurine Induces Measurable Changes of Short Latency Afferent Inhibition in a Group of Mild Cognitive Impairment Individuals. Frontiers in Aging Neuroscience, 6, 254. https://doi.org/10.3389/fnagi.2014.00254

11. Kocis, P., Tolar, M., Yu, J., Sinko, W., Ray, S. and Blennow, K. (2017) Elucidating the A $\beta 42$ Anti-Aggregation Mechanism of Action of Tramiprosate in Alzheimer's Disease: Integrating Molecular Analytical Methods, Pharmacokinetic and Clinical Data. CNS Drugs, 31, 495-509. https://doi.org/10.1007/s40263-017-0434-z

12. Ward, R., Cirkovic-Vellichovia, T., Ledeque, F., Tirizitis, G., Dubars, G. and Datla, K. (2006) Neuroprotection by Taurine and Taurine Analogues. In: Oja, S.S. and Saransaari, P., Eds., Taurine 6. Advances in Experimental Medicine and Biology, vol 583, Springer, Boston, MA, 299-306. https://doi.org/10.1007/978-0-387-33504-9_33

13. Roberti, G., Tanga, L., Michelessi, M., Quaranta, L., Parisi, V. and Manni, G. (2015) Cytidine 5'-Diphosphocholine (Citicoline) in Glaucoma: Rationale of Its Use, Current Evidence and Future Perspectives. International Journal of Molecular Sciences, 16, 28401-28417. https://doi.org/10.3390/ijms161226099

14. Pascolini, D. and Mariotti, S.P. (2012) Global Estimates of Visual Impairment: 2010. British Journal of Ophthalmology, 96, 614-618. https://doi.org/10.1136/bjophthalmol-2011-300539

15. Bourne, R.R.A., Jonas, J.B., Flaxman, S.R., Keeffe, J., Leasher, J. and Naidoo, K. (2014) Prevalence and Causes of Vision Loss in High-Income Countries and in Eastern and Central Europe: 1990-2010. British Journal of Ophthalmology, 98, 629-638. https://doi.org/10.1136/bjophthalmol-2013-304033

16. European Medicines Agency (EWP) (1997) Guideline on the Investigation of Bioequivalence Discussion in the Joint Efficacy and Quality Working Group. Adoption Rev. 1 by CHMP for Release for Consultation End of Consultation Rev. 1. 
17. Stahl, W., van den Berg, H., Arthur, J., Bast, A., Dainty, J. and Faulks, R.M. (2002) Bioavailability and Metabolism. Molecular Aspects of Medicine, 23, 39-100. https://doi.org/10.1016/S0098-2997(02)00016-X

18. Patterson, S.D., Zariffa, N.M., Montague, T.H. and Howland, K. (2001) Non-Traditional Study Designs to Demonstrate Average Bioequivalence for Highly Variable Drug Products. European Journal of Clinical Pharmacology, 57, 663-670. https://doi.org/10.1007/s002280100371

19. Jackson, A.J., Robbie, G. and Marroum, P. (2004) Metabolites and Bioequivalence: Past and Present. Clinical Pharmacokinetics, 43, 655-672. https://doi.org/10.2165/00003088-200443100-00002 